\title{
The Effect of Systemic Nitroglycerin Administration on the Kynurenine Pathway in the Rat
}

\author{
Gábor Nagy-Grócz ${ }^{1,2}$, Klaudia F. Laborc ${ }^{3}$, Gábor Veres ${ }^{1}$, Attila Bajtai $^{3}$, Zsuzsanna Bohár $^{1,3}$, \\ Dénes Zádori ${ }^{3}$, Annamária Fejes-Szabó ${ }^{1}$, Eleonóra Spekker ${ }^{3}$, László Vécsei ${ }^{1,3 *}$ and \\ Árpád Párdutz ${ }^{3}$
}

${ }^{1}$ MTA-SZTE Neuroscience Research Group, University of Szeged, Szeged, Hungary, ${ }^{2}$ Faculty of Health Sciences and Social Studies, University of Szeged, Szeged, Hungary, ${ }^{3}$ Department of Neurology, Faculty of Medicine, Albert Szent-Györgyi Clinical Center, University of Szeged, Szeged, Hungary

OPEN ACCESS

Edited by: Anna Andreou, Imperial College London, United Kingdom

Reviewed by: Marcelo M. Valença, Federal University of

Pernambuco, Brazil Stefan Evers,

Universität Münster, Germany

${ }^{*}$ Correspondence: László Vécsei vecsei.laszlo@med.u-szeged.hu

Specialty section: This article was submitted to Headache Medicine and Facial Pain, a section of the journal Frontiers in Neurology

Received: 15 February 2017 Accepted: 29 May 2017 Published: 14 June 2017

Citation:

Nagy-Grócz G, Laborc KF, Veres G,

Bajtai A, Bohár Z, Zádori D,

Fejes-Szabó A, Spekker E, Vécsei L and Párdutz Á (2017) The Effect of

Systemic Nitroglycerin Administration on the Kynurenine Pathway in the Rat.

Front. Neurol. 8:278.

doi: 10.3389/fneur.2017.00278
The primary headache disorders include migraine, which is one of the most frequent neurological disorders, which influences more than $14 \%$ of the whole population. Despite the research efforts, its exact pathomechanism is not fully revealed, but evidence points to the role of glutamate and its receptors. Kynurenic acid is an endogenous glutamate receptor antagonist produced by the kynurenine pathway (KP). Tryptophan 2,3-dioxygenase (TDO) and indoleamine 2,3-dioxygenase (IDO) convert L-tryptophan to $\mathrm{N}$-formylL-kynurenine, to be further transformed to L-kynurenine. Kynurenine aminotransferase-II (KAT-II), L-kynurenine hydrolase (KYNU), and L-kynurenine 3-monooxygenase (KMO) are key enzymes in the later steps of the KP. Nitroglycerin (NTG) administration serves as both human and animal model of migraine, causing the activation and sensitization in the trigeminal system. A previous study demonstrated a reduction of KAT-II expression following NTG administration in animals. The goal of current tests was to identify the potential modulatory effect of NTG on other metabolizing enzymes of the KP in the caudal trigeminal nucleus (TNC) of rats. Four hours following the intraperitoneal injection of NTG $(10 \mathrm{mg} / \mathrm{kg})$, the rats were perfused transcardially and the TNC was extracted for Western blotting. Western blot studies revealed that the expression of TDO2, IDO1, $\mathrm{KYNU}$, and KMO decreased in the TNC. The results demonstrated that NTG is able to downregulate the $\mathrm{KP}$, with a potential influence on the glutamatergic system as well, contributing to the development of trigeminal activation and sensitization in animals.

\section{Keywords: migraine, nitroglycerin, kynurenic acid, L-kynurenine, kynurenine pathway}

\section{INTRODUCTION}

Migraine is a common primary headache characterized by severe head pain and numerous concomitant symptoms, e.g., vomiting, nausea, photophobia, and phonophobia. The disease affects about $14 \%$ of the total population (1). The exact pathophysiology of the disorder is not fully understood, but it is

\footnotetext{
Abbreviations: GAPDH, glyceraldehyde 3-phosphate dehydrogenase; IDO, indoleamine 2,3-dioxygenase; KAT-II, kynurenine aminotransferase-II; KMO, L-kynurenine 3-monooxygenase; KYNA, kynurenic acid; KYNU, L-kynurenine hydrolase; KP, kynurenine pathway; L-KYN, L-kynurenine; NMDA, $N$-methyl-D-aspartate; NO, nitric oxide; NTG, nitroglycerin; PBS, phosphate-buffered saline; QUIN, quinolinic acid; TBST, Tris-buffered saline containing Tween 20; TDO, tryptophan 2,3-dioxygenase; TNC, caudal trigeminal nucleus; Trp, tryptophan.
} 
well known that the activation and sensitization of the trigeminal system is essential during the attack (2). Several lines of evidence have been put forth to support the hypothesis that glutamate receptors, principally $N$-methyl-D-aspartate (NMDA) receptors, have a pivotal aspect in these phenomena (3). Kynurenic acid (KYNA) is a neuroprotective metabolite that interacts with glutamate receptors, aryl hydrocarbon receptor, and G protein-coupled receptor 35 and elicits anti-glutamatergic actions. Series of data confirm that KYNA and its analogs have anti-nociceptive effects in several migraine models (4-6), probably by attenuating the trigeminal activation and sensitization. The initial process in the kynurenine pathway (KP) is the transformation of tryptophan (Trp) to $\mathrm{N}$-formyl-L-kynurenine by tryptophan 2,3-dioxygenase (TDO1, 2) and indoleamine 2,3-dioxygenase (IDO1,2): the rate-limiting enzymes of Trp metabolism. $N$-formyl-L-kynurenine is further converted by formamidase to L-kynurenine ( $\mathrm{L}-\mathrm{KYN})$, which is converted to KYNA by kynurenine aminotransferases (KATs), to 3-hydroxykynurenine by L-kynurenine 3-monooxygenase (KMO), and to anthranilic acid by L-kynurenine hydrolase (KYNU). The other metabolite of the KP is quinolinic acid (QUIN). In contrast to KYNA, QUIN is an agonist of the NMDA receptors and can provoke neuronal death and also causes lipid peroxidation and generates reactive oxygen species $(7,8)$ (Figure 1).

Systemic administration of nitroglycerin (NTG) can be utilized as a human and animal model of migraine. NTG is enzymatically converted to nitric oxide (NO) in the body, probably by a mitochondrial aldehyde dehydrogenase (9). The administration of NTG is able to activate and sensitize the trigeminal system in humans and animals (10-12). In our previous study, it was demonstrated that NTG decreased the expression of kynurenine aminotransferase-II (KAT-II) (13), which converts L-KYN to KYNA in the brain thus reducing KYNA levels, contributing to the hyperactivity of NMDA receptors.

The goal of this study was to explore the issue of NTG on the expression levels of TDO2, IDO1, KYNU, and KMO enzymes in the caudal trigeminal nucleus (TNC).

\section{MATERIALS AND METHODS}

\section{Animals}

We followed the directives for the Use of Animals in Research of the International Association for the Study of Pain and the policy of the European Economic Community (86/609/ECC). They were authorized by the local ethical committee of University of Szeged and the Scientific Ethics Committee for Animal Research of the Protection of Animals Advisory Board (XXIV./352/2012). 44 adult male Sprague-Dawley rats of 200-250 g bodyweight were used. The rodents were raised and maintained under standard laboratory conditions with tap water and regular rat chow available ad libitum on a $12 \mathrm{~h}$ dark-12 h light cycle.

\section{Drug Administration}

The animals were separated into two groups $(n=5)$. The animals in the first group, called placebo group, received only the vehicle solution (physiological saline) as treatment. In the second group, the rats were treated with an intraperitoneal injection of NTG (10 mg/kg bodyweight, Pohl Boskamp).

\section{Western Blot Analysis}

Four hours after the placebo/NTG injection, the animals were perfused transcardially with $100 \mathrm{~mL}$ phosphate-buffered saline and the dorsal horns of TNC segments $(+1$ and $-5 \mathrm{~mm}$ from the obex) were extracted. The samples were stored at $-80^{\circ} \mathrm{C}$ and they were sonicated in ice cold lysis buffer containing $50 \mathrm{mM}$ Tris- $\mathrm{HCl}$, $150 \mathrm{mM} \mathrm{NaCl}, 0.1 \%$ igepal, $0.1 \%$ cholic acid, $2 \mu \mathrm{g} / \mathrm{mL}$ leupeptin, $2 \mathrm{mM}$ phenylmethylsulfonyl fluoride, $1 \mu \mathrm{g} / \mathrm{mL}$ pepstatin, $2 \mathrm{mM}$ EDTA, and $0.1 \%$ sodium dodecyl sulfate. The homogenates were centrifuged for $10 \mathrm{~min}$ at $12,000 \mathrm{RPM}$ at $4^{\circ} \mathrm{C}$ and supernatants were aliquoted and stored at $-20^{\circ} \mathrm{C}$ until use. BCA Protein Assay Kit was used to measure protein concentration. Samples were mixed with sample buffer and were boiled for $3 \mathrm{~min}$. Standard SDS polyacrylamide gel electrophoresis was performed with equal amounts of protein samples $(20 \mu \mathrm{g} / \mathrm{lane})$ loaded on $10 \%$ Tris-glycine gel and electrotransferred onto Amersham Hybond-ECL nitrocellulose membrane $(0.45 \mu \mathrm{m}$ pore size). Page Ruler Prestained Protein Ladder $(10-170 \mathrm{kDa})$ was used to define approximate molecular weights. Non-specific binding was eliminated by blocking in Trisbuffered saline containing Tween 20 (TBST) and 5\% non-fat dry milk for $1 \mathrm{~h}$ at room temperature. Then, membranes were incubated in TBST containing 1\% non-fat dry milk and TDO antibody (LifeSpan BioSciences, LS-C111058, dilution: 1:500, incubation: overnight at room temperature), IDO antibody (Abcam, ab106134, dilution: 1:500, incubation: overnight at room temperature), KYNU antibody (Abcam, ab96365, dilution: 1:500, incubation: overnight at room temperature), KMO antibody (Abcam, ab83929, dilution: 1:4,000, incubation: overnight at room temperature), or glycerinaldehyde 3-phosphate dehydrogenase (GAPDH) antibody (Cell Signaling Technologies, 8884, dilution: 1:1,000, incubation: overnight at room temperature). On the following day, a horseradish peroxidase-conjugated anti-rabbit secondary antibody (Santa Cruz Biotechnology, sc-2030) in TBST containing 1\% non-fat dry milk was applied for $2 \mathrm{~h}$ at room temperature. SuperSignal West Pico Chemiluminescent Substrate was used to visualize bands on Carestream Kodak BioMax Light film. Western blot protocol was developed based on previous experiments $(4,14-16)$.

An observer blinded to the experimental groups carried out the measurements. The detailed methods were described previously (13).

Films were quantified by Java Image $1.47 \mathrm{v}$ analysis software (National Institutes of Health). The data were standardized to GAPDH.

\section{Statistical Analysis}

Statistical analysis was carried out by SPSS Statistics software (Version 20.0 for Windows, SPSS Inc.). Normality was checked by Kolmogorov-Smirnov test, and group means were compared by independent $t$-test, with $p<0.05$ taken as statistically significant. Group values are presented as means \pm SEM.

\section{RESULTS}

\section{NTG Induced a Decrease in TDO2 Expression in the TNC}

TDO2 protein was identified at $50 \mathrm{kDa}$ in Western blot assay. Densitometric analyses showed that the TDO2 bands were 


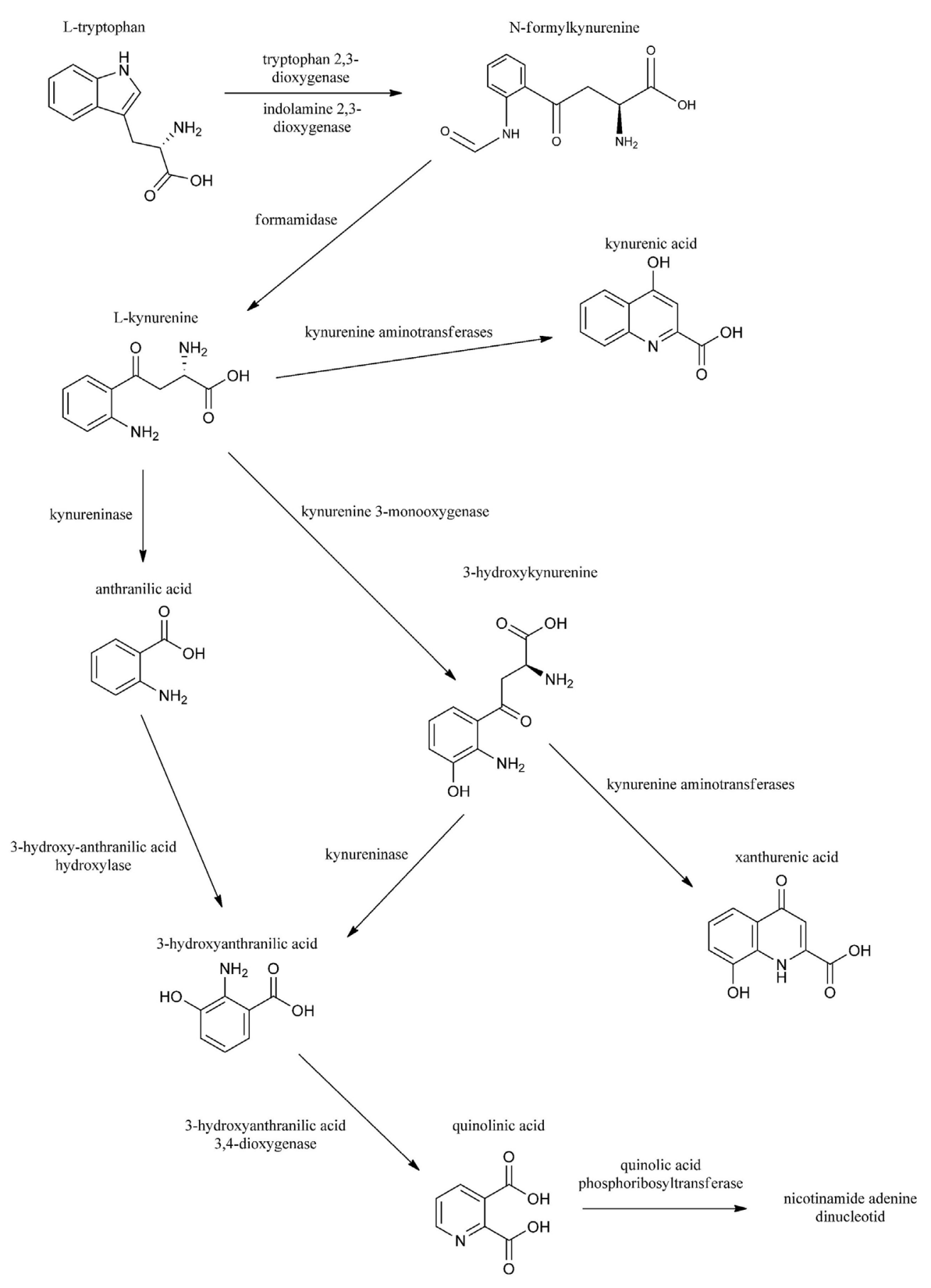

FIGURE 1 | The kynurenine pathway.

significantly decreased $(p<0.05)$ in the TNC after NTG administration as correlated with the placebo-treated animals (Figure 2).

\section{NTG Treatment Resulted in a Diminished ID01 Expression}

A band characteristic of the IDO1 protein was referred at $45 \mathrm{kDa}$ in Western blot assay. Densitometric analyses confirmed that the
IDO1 bands were significantly weaker $(p<0.05)$ in the TNC after NTG administration as correlated with the placebo-treated animals (Figure 3).

\section{NTG Was Able to Reduce the Expression of KYNU}

We could determine a band at $35 \mathrm{kDa}$ characteristic for the KYNU protein. In animals, which had received NTG, the density 

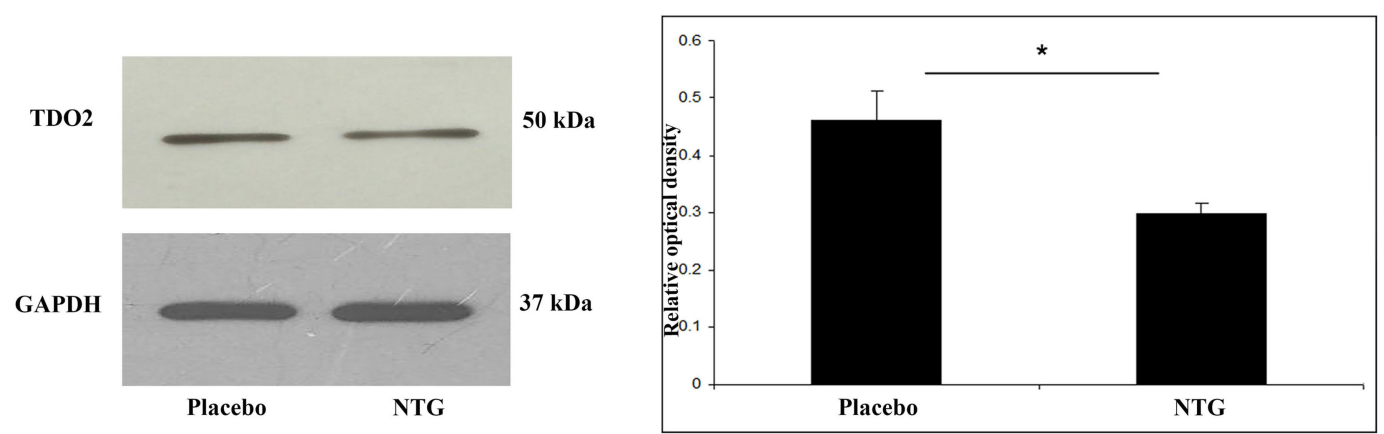

FIGURE 2 | Western blot analysis of TDO2 and GAPDH protein from the TNC. The quantitative analysis shows that in the NTG group the relative optical density of TDO2 specific bands were significantly less pronounced compared with the placebo group. ${ }^{*} p<0.05$; GAPDH, glyceraldehyde 3-phosphate dehydrogenase; NTG, nitroglycerin; TDO2, tryptophan 2,3-dioxygenase 2; TNC, caudal trigeminal nucleus.
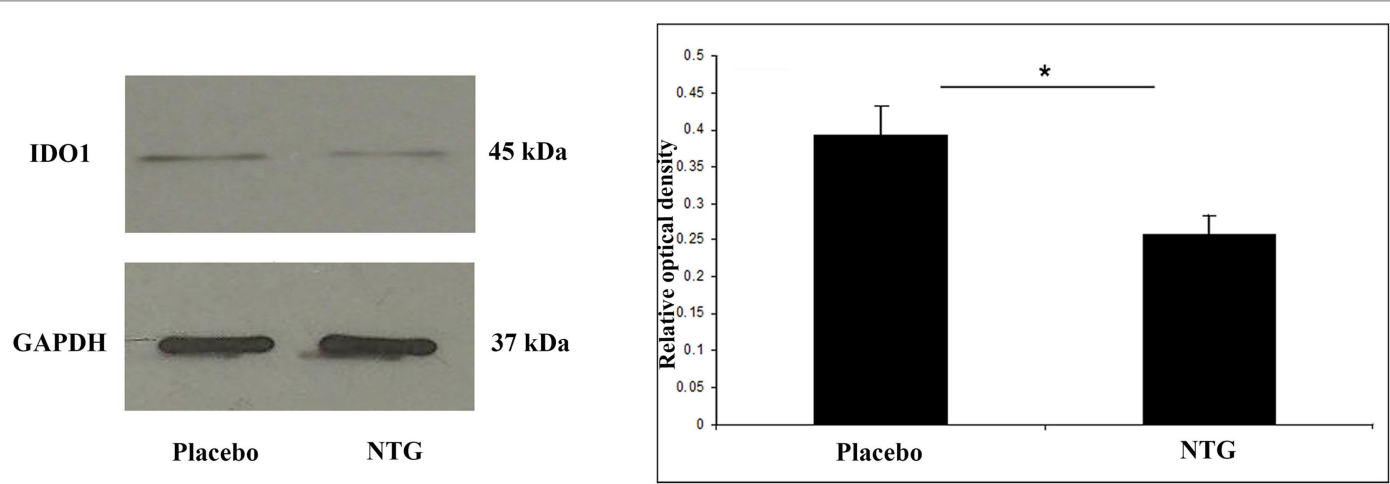

FIGURE 3 | Western blot of IDO1 and GAPDH expression in the TNC. The quantitative analysis shows that in the NTG group, the relative optical density of IDO1-specific bands was significantly decreased compared with the placebo group. ${ }^{*} p<0.05$; GAPDH, glyceraldehyde 3-phosphate dehydrogenase; IDO1, indoleamine 2,3-dioxygenase; NTG, nitroglycerin; TNC, caudal trigeminal nucleus.

of KYNU protein bands was weaker in TNC segments $(p<0.05)$ as compared with the placebo-treated group (Figure 4).

\section{KMO Expression Was Lower after NTG Administration}

L-Kynurenine 3-monooxygenase protein was identified at $56 \mathrm{kDa}$ in Western blot assay. Densitometric analyses showed that the KMO bands were significantly decreased $(p<0.05)$ in segments TNC after NTG administration as correlated with the placebotreated animals (Figure 5).

\section{DISCUSSION}

The current data demonstrated that NTG is able to decrease the expression of the KP enzymes in rat TNC. However, the question arises how NTG/NO can influence the KP. It is well known that the nitrergic system is able to alter the KP, e.g., NO can inhibit IDO expression via reversible binding to the active site in macrophages (17) and IDO activity is downregulated by NO production in bone marrow cells (18). The other hand, it is also known that the expression of $\mathrm{KMO}$ influences $\mathrm{NO}$ production in human HEK293 cells (19). Moreover, Backhaus and her colleagues showed in a mass spectrometry and NMR study that there is a direct interaction between kynurenine metabolites (e.g., 3-hydroxykynurenine and 3-hydroxyanthranilic acid) and NO (20).

It is also important to note that kynurenines have a crucial role in immune regulation (21). The transcriptional expression of IDO, KMO, and KYNU is also under the control of interferons (21), thus pro-inflammatory cytokines influence KP (22). Lögters and co-workers have shown that the ratio of kynureninetryptophan was increased in the blood of patients with posttraumatic sepsis (23), which pointed out that inflammation could modulate KP.

In this context, it is crucial that NO may cause neurogenic inflammation in the central nervous system. This is supported by observations that NTG was able to increase the expression of nuclear factor $\kappa \mathrm{B}$ in the trigeminocervical complex of rats $(13,24)$, which is a key player in the inflammation process controlled by cytokines. In human studies, Tfelt-Hansen and his group demonstrated that infusion of NTG can trigger inflammatory 


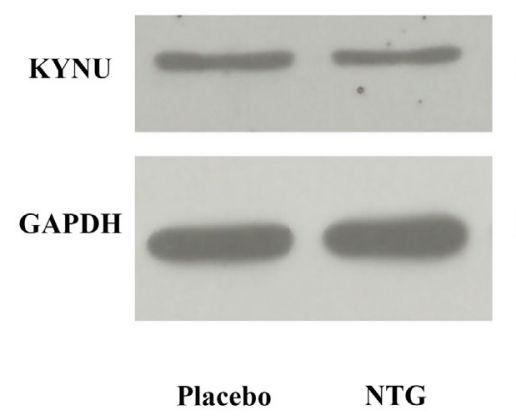

$35 \mathrm{kDa}$

$37 \mathrm{kDa}$

Placebo NTG

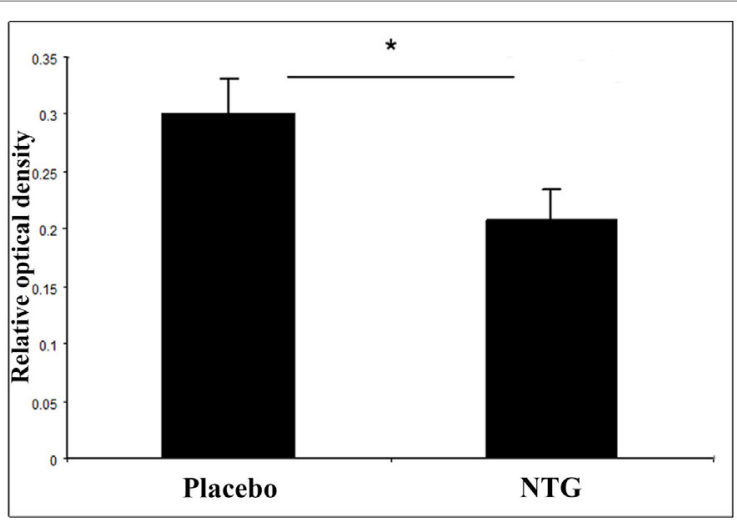

FIGURE 4 | Representative Western blot bands and diagram of KYNU and GAPDH in the TNC. The quantitative analysis shows that in the NTG group, the relative optical density of KYNU-specific bands were significantly smaller compared with the placebo group. ${ }^{*} p<0.05$; GAPDH, glyceraldehyde 3-phosphate dehydrogenase; KYNU, kynurenine hydrolase; NTG, nitroglycerin; TNC, caudal trigeminal nucleus.

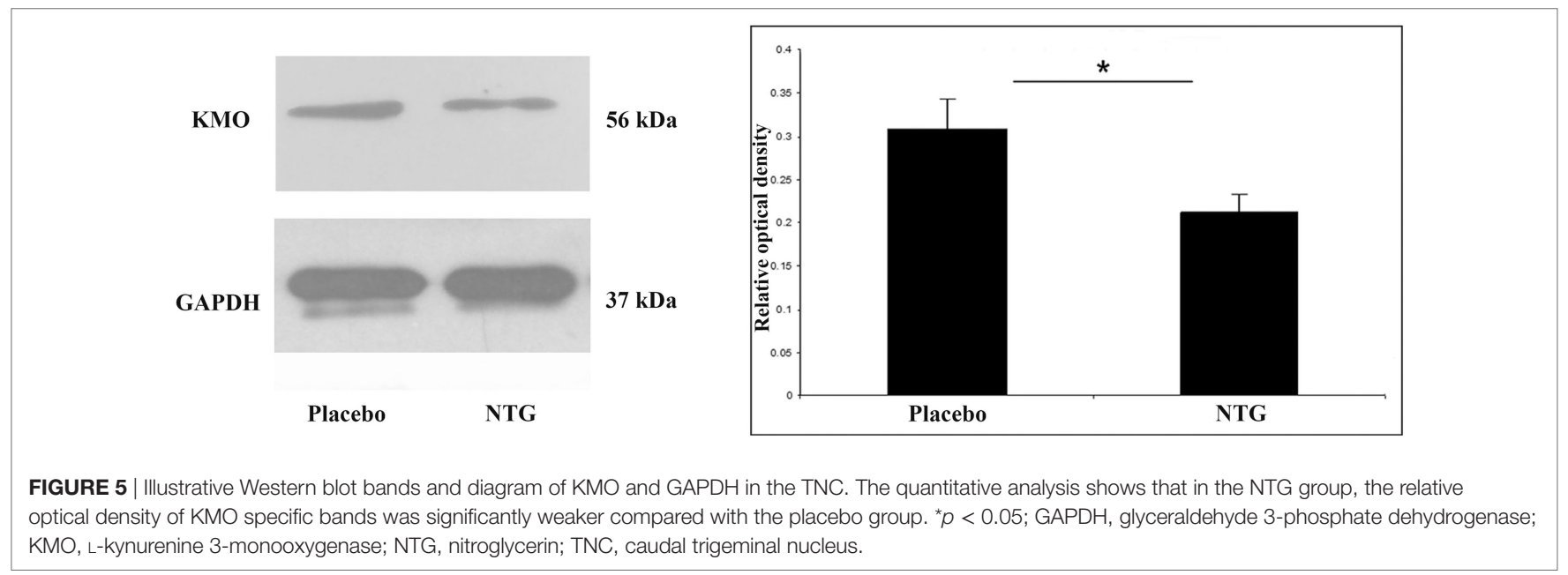

response by inducing inflammatory mediators, which response was inhibited by the anti-inflammatory drug, prednisolone (25).

In the previous study, we demonstrated that NTG was able to decrease the expression of KAT-II, which produces KYNA. Our present and earlier Western blot data showed that NTG could reduce the expression levels of the KP enzymes.

Our findings are comparable with recent studies, which showed that the chronic migraine and cluster headaches are associated with altered levels of kynurenine metabolites, i.e., reduced levels of KYNA and L-KYN have been found in the serum of these patients $(26,27)$. These findings are in accordance with the theory of an increased release of glutamate probably yielding to a hyperactivity of glutamate receptors.

Migraine can be characterized by an increase in glutamatergic function (28), yielding fully activated NMDA receptors by the high glutamate levels, which might combine with small KYNA levels. Increased glutamate levels were found in the human cerebrospinal fluid, platelets, and plasma of migraine sufferers $(29,30)$.
To summarize the human and animal data, we can conclude that the KP is downregulated under the different types of headaches and thus possibly providing less KYNA. These data are in line with the theory of hyperactive NMDA receptors having a crucial role in the migraine pathophysiology. These receptors are key players in the mechanism of central sensitization (31), which have a pivotal role in the pathophysiology of migraine. Our present data strongly confirm that the KP has a relevant role in the pathomechanism of the trigeminal activation and sensitization, thus in the migraine pathology as well. In summary, influencing the KP provides a possible new target in the future therapy of migraine.

\section{ETHICS STATEMENT}

We followed the directives for the Use of Animals in Research of the International Association for the Study of Pain and the policy of the European Economic Community (86/609/ECC). They were authorized by the local ethical committee of University of 
Szeged and the Scientific Ethics Committee for Animal Research of the Protection of Animals Advisory Board (XXIV./352/2012).

\section{AUTHOR CONTRIBUTIONS}

GN-G: participated in the design and implementation of experiments, statistical analysis, interpreted data, and wrote the manuscript. KL, GV, AB, ZB, DZ, AF-S, and ES: participated in the implementation of the experiments and statistical analysis. LV: participated in the design of the experiments and in the final approval of the version to be published. ÁP: participated in the conception and design of the experiments and in the interpretation of the data and writing. All authors: critical revision of the manuscript.

\section{REFERENCES}

1. Stovner LJ, Andree C. Prevalence of headache in Europe: a review for the Eurolight project. J Headache Pain (2010) 11(4):289-99. doi:10.1007/ s10194-010-0217-0

2. Edvinsson L, Uddman R. Neurobiology in primary headaches. Brain Res Brain Res Rev (2005) 48(3):438-56. doi:10.1016/j.brainresrev.2004. 09.007

3. Woolf CJ, Salter MW. Neuronal plasticity: increasing the gain in pain. Science (2000) 288(5472):1765-9. doi:10.1126/science.288.5472.1765

4. Fejes-Szabo A, Bohar Z, Vamos E, Nagy-Grocz G, Tar L, Veres G, et al. Pre-treatment with new kynurenic acid amide dose-dependently prevents the nitroglycerine-induced neuronal activation and sensitization in cervical part of trigemino-cervical complex. J Neural Transm (Vienna) (2014) 121(7):725-38. doi:10.1007/s00702-013-1146-2

5. Csati A, Edvinsson L, Vecsei L, Toldi J, Fulop F, Tajti J, et al. Kynurenic acid modulates experimentally induced inflammation in the trigeminal ganglion. J Headache Pain (2015) 16:99. doi:10.1186/s10194-015-0581-x

6. Lukacs M, Warfvinge K, Kruse LS, Tajti J, Fulop F, Toldi J, et al. KYNA analogue SZR72 modifies CFA-induced dural inflammation-regarding expression of pERK1/2 and IL-1beta in the rat trigeminal ganglion. J Headache Pain (2016) 17(1):64. doi:10.1186/s10194-016-0654-5

7. Behan WM, McDonald M, Darlington LG, Stone TW. Oxidative stress as a mechanism for quinolinic acid-induced hippocampal damage: protection by melatonin and deprenyl. Br J Pharmacol (1999) 128(8):1754-60. doi:10.1038/ sj.bjp. 0702940

8. Guidetti P, Schwarcz R. 3-Hydroxykynurenine potentiates quinolinate but not NMDA toxicity in the rat striatum. Eur J Neurosci (1999) 11(11):3857-63. doi:10.1046/j.1460-9568.1999.00806.x

9. Chen Z, Foster MW, Zhang J, Mao L, Rockman HA, Kawamoto T, et al. An essential role for mitochondrial aldehyde dehydrogenase in nitroglycerin bioactivation. Proc Natl Acad Sci U S A (2005) 102(34):12159-64. doi:10.1073/ pnas.0503723102

10. Tassorelli C, Joseph SA. NADPH-diaphorase activity and Fos expression in brain nuclei following nitroglycerin administration. Brain Res (1995) 695(1):37-44. doi:10.1016/0006-8993(95)00732-6

11. Tassorelli C, Joseph SA. Systemic nitroglycerin induces Fos immunoreactivity in brainstem and forebrain structures of the rat. Brain Res (1995) 682(1-2):167-81. doi:10.1016/0006-8993(95)00348-T

12. Di Clemente L, Coppola G, Magis D, Gerardy PY, Fumal A, De Pasqua V, et al. Nitroglycerin sensitises in healthy subjects CNS structures involved in migraine pathophysiology: evidence from a study of nociceptive blink reflexes and visual evoked potentials. Pain (2009) 144(1-2):156-61. doi:10.1016/j. pain.2009.04.018

13. Nagy-Grocz G, Tar L, Bohar Z, Fejes-Szabo A, Laborc KF, Spekker E, et al. The modulatory effect of anandamide on nitroglycerin-induced sensitization in the trigeminal system of the rat. Cephalalgia (2016) 36(9):849-61. doi: $10.1177 / 0333102415613766$

\section{ACKNOWLEDGMENTS}

This work was supported by the Hungarian Brain Research Program (Grant No. KTIA_13_NAP-A-III/9), the EUROHEADPAIN (FP7-Health 2013-Innovation; Grant No. 602633), and GINOP2.3.2-15-2016-00034. Methods section is developed based on previous experiments, which is already present in theses and is in line with the author's university policy, and can be accessed online. GN-G was supported through the UNKPÚNKP-16-3 New National Excellence Program of the Ministry of Human Capacities. ÁP and DZ were supported by the Bolyai Scholarship Programme of the Hungarian Academy of Sciences. We are indebted to Mrs. Valéria Vékony for histotechnical assistance.

14. Fejes-Szabo A. Modulatory Effect of Synthetic Kynurenic Acid Derivatives on the Nitroglycerin-Induced Trigeminal Activation and Sensitization in Rats [Dissertation]. University of Szeged (2014).

15. Fejes-Szabo A, Bohar Z, Nagy-Grocz G, Vamos E, Tar L, Podor B, et al. Effect of probenecid on the pain-related behaviour and morphological markers in orofacial formalin test of the rat. CNS Neurol Disord Drug Targets (2015) 14(3):350-9. doi:10.2174/1871527314666150225141229

16. Bohár Z. Activation Pattern and Modulation of Pain Related Structures in Animal Models of Migraine [Dissertation]. Universtiy of Szeged (2016).

17. Thomas SR, Terentis AC, Cai H, Takikawa O, Levina A, Lay PA, et al. Posttranslational regulation of human indoleamine 2,3-dioxygenase activity by nitric oxide. J Biol Chem (2007) 282(33):23778-87. doi:10.1074/jbc.M700669200

18. Hara T, Ogasawara N, Akimoto H, Takikawa O, Hiramatsu R, Kawabe T, et al. High-affinity uptake of kynurenine and nitric oxide-mediated inhibition of indoleamine 2,3-dioxygenase in bone marrow-derived myeloid dendritic cells. Immunol Lett (2008) 116(1):95-102. doi:10.1016/j.imlet.2007. 11.016

19. Wilson K, Auer M, Binnie M, Zheng X, Pham NT, Iredale JP, et al. Overexpression of human kynurenine-3-monooxygenase protects against 3-hydroxykynurenine-mediated apoptosis through bidirectional nonlinear feedback. Cell Death Dis (2016) 7:e2197. doi:10.1038/cddis.2016.87

20. Backhaus C, Rahman H, Scheffler S, Laatsch H, Hardeland R. NO scavenging by 3-hydroxyanthranilic acid and 3-hydroxykynurenine: $\mathrm{N}$-nitrosation leads via oxadiazoles to o-quinone diazides. Nitric Oxide (2008) 19(3):237-44. doi:10.1016/j.niox.2008.07.002

21. Mandi Y, Vecsei L. The kynurenine system and immunoregulation. J Neural Transm (Vienna) (2012) 119(2):197-209. doi:10.1007/s00702-011-0681-y

22. Hassanain HH, Chon SY, Gupta SL. Differential regulation of human indoleamine 2,3-dioxygenase gene expression by interferons-gamma and -alpha. Analysis of the regulatory region of the gene and identification of an interferongamma-inducible DNA-binding factor. J Biol Chem (1993) 268(7):5077-84.

23. Lögters TT, Laryea MD, Altrichter J, Sokolowski J, Cinatl J, Reipen J, et al. Increased plasma kynurenine values and kynurenine-tryptophan ratios after major trauma are early indicators for the development of sepsis. Shock (2009) 32(1):29-34. doi:10.1097/SHK.0b013e31819714fa

24. Greco R, Tassorelli C, Cappelletti D, Sandrini G, Nappi G. Activation of the transcription factor NF-kappaB in the nucleus trigeminalis caudalis in an animal model of migraine. Neurotoxicology (2005) 26(5):795-800. doi:10.1016/j. neuro.2005.02.005

25. Tfelt-Hansen P, Daugaard D, Lassen LH, Iversen HK, Olesen J. Prednisolone reduces nitric oxide-induced migraine. Eur J Neurol (2009) 16(10):1106-11. doi:10.1111/j.1468-1331.2009.02654.x

26. Curto M, Lionetto L, Negro A, Capi M, Fazio F, Giamberardino MA, et al. Altered kynurenine pathway metabolites in serum of chronic migraine patients. J Headache Pain (2015) 17:47. doi:10.1186/s10194-016-0638-5

27. Curto M, Lionetto L, Negro A, Capi M, Perugino F, Fazio F, et al. Altered serum levels of kynurenine metabolites in patients affected by cluster headache. J Headache Pain (2015) 17:27. doi:10.1186/s10194-016-0620-2 
28. Vecsei L, Szalardy L, Fulop F, Toldi J. Kynurenines in the CNS: recent advances and new questions. Nat Rev Drug Discov (2013) 12(1):64-82. doi:10.1038/nrd3793

29. Cananzi AR, D’Andrea G, Perini F, Zamberlan F, Welch KM. Platelet and plasma levels of glutamate and glutamine in migraine with and without aura. Cephalalgia (1995) 15(2):132-5. doi:10.1046/j.1468-2982.1995.015002132.x

30. Peres MF, Zukerman E, Senne Soares CA, Alonso EO, Santos BF, Faulhaber MH. Cerebrospinal fluid glutamate levels in chronic migraine. Cephalalgia (2004) 24(9):735-9. doi:10.1111/j.1468-2982.2004.00750.x

31. Sarchielli P, Di Filippo M, Nardi K, Calabresi P. Sensitization, glutamate, and the link between migraine and fibromyalgia. Curr Pain Headache Rep (2007) 11(5):343-51. doi:10.1007/s11916-007-0216-2
Conflict of Interest Statement: The authors declare that the research was conducted in the absence of any commercial or financial relationships that could be construed as a potential conflict of interest.

Copyright (๑ 2017 Nagy-Grócz, Laborc, Veres, Bajtai, Bohár, Zádori, Fejes-Szabó, Spekker, Vécsei and Párdutz. This is an open-access article distributed under the terms of the Creative Commons Attribution License (CC BY). The use, distribution or reproduction in other forums is permitted, provided the original author(s) or licensor are credited and that the original publication in this journal is cited, in accordance with accepted academic practice. No use, distribution or reproduction is permitted which does not comply with these terms. 